\title{
Detección espectrofotométrica de sobredosis de monensina en piensos para bovinos
}

\author{
Berneri, M.J. ${ }^{1,3}$; Miranda, A.O. ${ }^{1}$; Frances, O. ${ }^{2}$; Mastrantonio, G.E. ${ }^{3}$ \\ ${ }^{1}$ Área Salud Pública Veterinaria EEA INTA Anguil, La Pampa, Argentina. ${ }^{2}$ Cátedra Clín.Grandes Anim. \\ Fac. Cs. Vet. UNLPam, Gral. Pico, La Pampa, Argentina. ${ }^{3}$ Área Toxicología, Fac. Cs. Exactas UNLP, \\ La Plata, Argentina. E-mail: mastra@biol.unlp.edu.ar
}

\begin{abstract}
Berneri, M.J.; Miranda, A.O.; Frances, O.; Mastrantonio, G.E.: Detección espectrofotométrica de sobredosis de monensina en piensos para bovinos. Rev. vet. 27: 1, 32-35, 2016. La creciente presión de la agricultura, así como la búsqueda de mayor eficiencia en los sistemas ganaderos, ha conducido al aumento del empleo de productos farmacológicos como estrategias para mejorar la rentabilidad de la ganadería. La monensina (MN) es un antibiótico poliéter del grupo de los ionóforos elaborado a partir del hongo Streptomyces cinnamonensis. En este trabajo se describe la puesta a punto de un método espectrofotométrico sencillo y eficaz para la determinación de $\mathrm{MN}$ en muestras de piensos para ganado bovino. Se evaluaron alimentos con diferentes concentraciones de MN conocidas e incógnitas, recolectadas en las provincias de Córdoba y La Pampa (Argentina). Los resultados muestran que el método espectrofotométrico es apropiado para medir $\mathrm{MN}$ en piensos a dosis normales y en sobredosificaciones, siendo útil para revelar presuntas intoxicaciones de origen alimentario. Comparada con otros métodos, la técnica revela bajo costo en insumos y equipamiento, aunque requiere cierta laboriosidad. Los resultados del trabajo señalan la idoneidad del método para efectuar la vigilancia farmacológica de $\mathrm{MN}$, dado que brinda un rápido y efectivo diagnóstico en casos de intoxicación, de gran utilidad en laboratorios de baja complejidad.
\end{abstract}

Palabras clave: bovino, alimento, monensina, intoxicación, espectrofotometría.

\begin{abstract}
Berneri, M.J.; Miranda, A.O.; Frances, O.; Mastrantonio, G.E.: Spectrophotometric detection of monensin overdose in bovine feed. Rev. vet. 27: 1, 32-35, 2016. The growing pressure on agriculture as well as the search for better efficiency in livestock systems has led to the increased use of pharmacological products as a strategy to improve livestock profitability. Monensin (MN) is a polyether antibiotic of the ionophores class produced by the Streptomyces cinnamonensis. This study describes the development of a simple and effective spectrophotometric method for MN measurement in feed samples. Feed samples with known and unknown concentrations of MN collected from farms of Córdoba and La Pampa provinces (Argentina) were measured. The results show that the spectrophotometric method is appropriate for measuring normal and overdose levels of $\mathrm{MN}$ in feed. Thus, the method is suitable to evaluate presumptive poisoning cases. While reagents and equipment are of low cost compared to more sophisticated techniques, it does require more labor and time. We suggest the use of spectrophotometric method for monitoring and eventual quick diagnosis of poisoning cases, being a useful tool for low complexity laboratories.
\end{abstract}

Key words: cattle, feed, monensin, intoxication, spectrophotometry.

\section{INTRODUCCIÓN}

La creciente presión de la agricultura, así como la búsqueda de una mayor eficiencia en los sistemas ganaderos, ha conducido a un mayor uso de herramientas farmacológicas como estrategias para incrementar la rentabilidad. En ese contexto, el uso de antibióticos ha generado una mejora sustancial de la eficiencia en los sistemas de engorde, hasta alcanzar un uso casi masivo a lo largo de los últimos años ${ }^{2}$. Uno de ellos es la monensina $(\mathrm{MN})$, un antibiótico del tipo poliéter $(\mathrm{PE})$ con actividad como ionóforo, elaborado por el hongo Streptomyces cinnamonensis, utilizado desde hace más 
de tres décadas como regulador del metabolismo ruminal ${ }^{1}$.

El uso de $\mathrm{MN}$ se ha incrementado en los últimos años, al descubrirse sus efectos benéficos sobre la cría vacuna, así como la optimización de la conversión de alimentos y la prevención de patologías como meteorismo, acidosis y coccidiosis bovina. La dosis terapéutica establecida para $\mathrm{MN}$ es de 1 a $3 \mathrm{mg} / \mathrm{kg}$ de peso vivo (equivalente a $30 \mathrm{mg} / \mathrm{kg}$ de alimento). No obstante, con frecuencia se reportan intoxicaciones a causa del uso de formulaciones inadecuadas, incorrecta preparación en la pre-mezcla, fallas en el mezclado y mala distribución en el comedero ${ }^{7}$.

Por estas razones, la búsqueda de un método de cuantificación de $\mathrm{MN}$ en piensos, utilizando equipamientos y métodos al alcance de los veterinarios de la especialidad, constituye un objetivo de gran aplicabilidad en producción animal. En el presente trabajo se describe un método analítico concebido para ser desarrollado en laboratorios de baja complejidad y se evalúa su desempeño en diferentes matrices alimentarias.

\section{MATERIAL Y MÉTODOS}

Técnica propuesta. Se empleó una modificación del método reportado por el Centro de Inspección de Alimentos y Materiales Agrícolas (FAMIC: Food and Agricultural Materials Inspection Center, Tokyo, Japon) ${ }^{4}$. Tal institución recomienda un proceso que incluye dos etapas, que aquí denominaremos "pre-analítica" y "analítica". La primera incluye la preparación de la muestra, la obtención de un extracto y la derivatización por copulación con el cromóforo p-dimetil-amino-benzaldehído (PDB). La segunda fase es la determinación espectrofotométrica del producto de la copulación del compuesto, que varía dentro de la escala del color azul, con máxima absorbancia a $578 \mathrm{~nm}$.

Toma de muestras. Se analizaron muestras que contenían diferentes concentraciones de MN conocidas e incógnitas, recolectadas en las provincias de La Pampa y Córdoba, Argentina. Se procesaron diez muestras correspondientes a raciones administradas a bovinos en establecimientos con y sin problemas de intoxicación, así como también muestras de núcleos minerales y vitamínicos con el aditivo de MN. En aquellos casos en los cuales se reportó intoxicación alimentaria, se tomaron cinco alícuotas de distintos lugares del comedero, con posterior homogeneización de las mismas para conformar un volumen final de 500 g. Posteriormente se realizó el muestreo por cuarteo ${ }^{5}$ y se obtuvo una alícuota final de $100 \mathrm{~g}$.

Procesamiento de la muestra. Las muestras fueron procesadas en un molinillo para reducirlas a un polvo fino. Diez gramos de este material triturado fueron disueltos en $100 \mathrm{ml}$ de etanol anhidro, agitando la mezcla durante 20 minutos para promover una completa homogeneización. Luego, para favorecer la disolución, se sometió la mezcla a agitación/vibración en un baño ultrasónico durante 5 minutos y se filtró para obtener un extracto etanólico. Considerando que el medio acuoso interfiere en la reacción de copulación, todo el proceso se desarrolló evitando la contaminación con agua (reactivos, material de vidrio, operaciones de trasvase). Para establecer la curva de calibración se dispuso de un pienso como matriz de referencia. A partir de ella se preparó una muestra de referencia por incorporación de cantidades apropiadas de MN de calidad analítica. Se prepararon dos extractos etanólicos, uno desde la matriz de referencia (extracto A) y otro desde la muestra de referencia (extracto B). A partir de estos dos extractos se preparó una serie de diluciones en el rango de 0 a $110 \mathrm{mg} \mathrm{MN} / \mathrm{kg}$ de alimento con cinco niveles, por triplicado.

Reacción de derivatización. La reacción se efectuó en medio ácido, garantizado por la adición de $\mathrm{H}_{2} \mathrm{SO}_{4}$. El reactivo derivatizante fue el PDB, que generó un producto de color azul, con un máximo de absorción a $578 \mathrm{~nm}$. Se preparó PDB en etanol anhidro $(1,2 \mathrm{mg} /$ $\mathrm{ml}$ ), con un contenido final de $1 \%$ de $\mathrm{H}_{2} \mathrm{SO}_{4} 98 \%$. Esta solución fue preparada el mismo día de trabajo (no puede almacenarse). Se dispuso de una batería de tubos de ensayo por triplicado, en cada uno de los cuales se colocaron $3 \mathrm{ml}$ de la matriz de referencia (extracto A) con la concentración de $\mathrm{MN}$ correspondiente a cada punto de la curva, más $1 \mathrm{ml}$ de la solución de PDB. Todos los tubos fueron tapados e incubados en baño termostático a $70^{\circ} \mathrm{C}$ durante 20 min para el desarrollo de la reacción.

Espectrofotometría. Una vez frías, las soluciones se analizaron en un espectrofotómetro a $578 \mathrm{~nm}$, utilizando como blanco de reactivo una solución de etanol: $\mathrm{H}_{2} \mathrm{SO}_{4} 98 \%$. En las muestras que registraron concentraciones superiores a las del rango establecido por la curva de calibración, las lecturas fueron repetidas previa dilución al 1/2 con el extracto A.

Patrón secundario. El patrón de referencia para la puesta a punto del método fue una sal sódica de MN (Sigma-Aldrich). Para establecer la curva de calibración se utilizó un extracto obtenido por cristalización proveniente de concentrados minerales comerciales de $\mathrm{MN}$ de uso veterinario. Esta MN fue comparada mediante espectroscopía infrarroja con la sustancia de referencia, y debió demostrar características analíticas equivalentes a las obtenidas con el patrón comercial.

\section{RESULTADOS}

En la Tabla 1 se presentan los datos correspondientes a la curva de calibración de $\mathrm{MN}$ ajustada por regresión lineal. En la Tabla 2 se detallan las muestras analizadas y la composición de cada una de ellas, así como los resultados esperados y obtenidos.

En piensos formulados bajo diferentes condiciones (no asociados a casos de intoxicación) se registraron valores en un rango que iba desde el límite de detección de la técnica hasta $19 \mathrm{ppm}$. En muestras obtenidas en casos de intoxicación se hallaron valores que se ubicaron por debajo del límite de detección hasta niveles de 142 ppm. En muestras provenientes de núcleos comer- 
Tabla 1. Evaluación estadística del método analítico.

\begin{tabular}{ll}
\hline parámetros & valores \\
\hline número de muestras utilizadas & 25 \\
niveles & 6 \\
$\mathrm{r}^{2}$ & 0,99173 \\
ordenada al origen (error estadístico) & $0,13995 \pm 0,02241$ \\
pendiente & $0,01691\left(3,21887 \times 10^{-4}\right)$ \\
límite de detección (LOD) & $3 \mathrm{mg} / \mathrm{kg}$ \\
límite de cuantificación (LOQ) & $8 \mathrm{mg} / \mathrm{kg}$ \\
rango lineal & $8 \mathrm{a} 110 \mathrm{mg} \mathrm{MN} / \mathrm{kg}$ de alimento \\
rango normal (de decisión) & $8-40 \mathrm{mg} \mathrm{MN} / \mathrm{kg}$ de alimento \\
valor de riesgo veterinario & $200-300 \mathrm{mg} / \mathrm{kg}$ \\
\hline
\end{tabular}

LOD: límite de detección, LOQ: límite de cuantificación.

Tabla 2. Concentración de monensina esperada versus hallada en distintas muestras de alimentos.

\begin{tabular}{|c|c|c|c|c|}
\hline $\mathrm{N}^{\mathrm{o}}$ & descripción & composición de la muestra analizada & $\begin{array}{c}\mathrm{RE} \\
(\mathrm{mg} / \mathrm{kg})\end{array}$ & $\begin{array}{c}\mathrm{RH} \\
(\mathrm{mg} / \mathrm{kg})\end{array}$ \\
\hline 1 & $\begin{array}{l}\text { ración } \\
\text { normal }\end{array}$ & $\begin{array}{l}\text { Ración bovinos de carne (grano de maíz, pellet } \\
\text { de girasol, etc.) con incorporación de MN }\end{array}$ & 30 & $<\mathrm{LOQ}$ \\
\hline 2 & $\begin{array}{l}\text { ración } 1,5 \\
\text { veces la dosis }\end{array}$ & $\begin{array}{l}\text { Ración bovinos de carne (grano de maíz, pellet } \\
\text { de girasol, etc.) con incorporación de MN. }\end{array}$ & 45 & 8 \\
\hline 5 & $\begin{array}{l}\text { ración } \\
\text { normal }\end{array}$ & $\begin{array}{l}\text { Ración bovinos de carne } \\
\text { (grano de maíz, pellets de girasol). }\end{array}$ & 30 & $<\mathrm{LOD}$ \\
\hline 6 & $\begin{array}{l}\text { ración } \\
\text { normal }\end{array}$ & $\begin{array}{l}\text { Ración bovinos de carne } \\
\text { (grano de maíz, pellets de girasol). }\end{array}$ & 30 & 19 \\
\hline 3 & $\begin{array}{l}\text { caso } \\
\text { intoxicación }\end{array}$ & $\begin{array}{l}\text { Ración bovinos de carne } \\
\text { (grano de maíz, pellets de girasol). }\end{array}$ & 30 & $<\mathrm{LOQ}$ \\
\hline 4 & $\begin{array}{l}\text { caso } \\
\text { intoxicación }\end{array}$ & $\begin{array}{l}\text { Ración bovinos de carne } \\
\text { (grano de maíz, pellets de girasol). }\end{array}$ & 30 & $<\mathrm{LOD}$ \\
\hline 7 & $\begin{array}{l}\text { caso } \\
\text { intoxicación }\end{array}$ & $\begin{array}{l}\text { Ración bovinos de carne (grano de maíz, } \\
\text { pellets de girasol) Villa María, Córdoba. }\end{array}$ & 30 & 80 \\
\hline 8 & $\begin{array}{l}\text { caso } \\
\text { intoxicación }\end{array}$ & $\begin{array}{l}\text { Ración bovinos de carne } \\
\text { (grano de maíz, pellets de girasol). }\end{array}$ & 30 & 142 \\
\hline 9 & $\begin{array}{l}\text { núcleo } \\
\text { comercial }\end{array}$ & $\begin{array}{l}\text { Núcleo mineral bovinos con } \mathrm{MN} \\
\text { (para agregar al 10\%). }\end{array}$ & 300 & 23 \\
\hline 10 & $\begin{array}{l}\text { núcleo } \\
\text { comercial }\end{array}$ & $\begin{array}{l}\text { Núcleo mineral bovinos con } \mathrm{MN} \\
\text { (para agregar al 10\%). }\end{array}$ & 300 & 46 \\
\hline
\end{tabular}

RE: resultado esperado en alimento en virtud del procedimiento de dosificación y mezcla informado; RH: resultado hallado en alimento, LOD: límite de detección, LOQ: límite de cuantificación

ciales, donde se esperaban resultados 10 veces mayores a los del pienso (300 ppm) se hallaron niveles máximos de 46 ppm.

\section{DISCUSIÓN}

En la región denominada "pampa húmeda" de Argentina, se han reportado varios incidentes aislados, relacionados con la intoxicación del ganado por sobredosificación con MN. En ninguno de dichos casos se realizó la determinación de la concentración de $\mathrm{MN}$ en los piensos involucrados. Ello fue así fundamentalmente porque no existen protocolos de preparación de piensos adicionados con premezclas conteniendo $\mathrm{MN}$, destinados al control de las concentraciones finales del antibiótico. Tal afirmación se basa en nuestras experiencias adquiridas en el campo y coinciden con afirmaciones publicadas por otros colegas ${ }^{10}$.
La MN posee una gran afinidad por cationes como el sodio, lo cual altera in vivo la polaridad de la membrana celular, afectando las fibras de los músculos esquelético y cardíaco, generando una miocardiopatía que cursa con estasis sanguínea y consecuente edema intersticial pulmonar ${ }^{8}$. La toxicidad puede presentarse tanto en forma aguda como crónica, dependiendo de la dosis ingerida. $\mathrm{La} \mathrm{DL}_{50}$ en vacunos se ha establecido entre 50 y $80 \mathrm{mg} / \mathrm{kg}$ de peso vivo, en tanto que las primeras manifestaciones clínicas de intoxicación aparecen frecuentemente con dosis de $10 \mathrm{mg} / \mathrm{kg}$ de peso vivo ${ }^{9}$.

Ello implicaría un estrecho margen para las sobredosis, con elevado riesgo de muerte del animal intoxicado. Sin embargo, las tasas de mortalidad reportadas han sido muy variables con valores que oscilan entre $1 \%$ y $26 \%{ }^{7,10,11}$. La ausencia de acuerdo respecto a los márgenes de seguridad de $\mathrm{MN}$ puede ser una de las causas que está generando un creciente aumento de casos de intoxicación en bovinos bajo alimentación controlada.

A comienzos de la década de 1970 se establecieron varios métodos analíticos de detección y confirmación de uno o más antibióticos del tipo PE en alimentos, tal como la MN. La mayoría de tales métodos se basan en cromatografía líquida de alta presión (HPLC), lo cual requiere un equipamiento de laboratorio de alta complejidad y -consecuentemente- elevado costo. Dado que varios de estos PE no responden al ultravioleta o a la fluorescencia, los procedimientos analíticos deben recurrir a pasos previos denominados "derivatizaciones" o al uso de detectores de masa, lo cual torna más complejas las mediciones ${ }^{3,4,6}$.

En cuanto al método aquí descrito, aunque parcialmente laborioso, permite obtener resultados certeros dentro de los rangos de interés esperados. La variabilidad en cuanto a los resultados obtenidos, no solo para aquellos casos en los cuales la concentración de $\mathrm{MN}$ se encontraba dentro de los límites terapéuticos, sino también para aquellas muestras correspondientes a casos de intoxicación, pudo ser ocasionada por fallas del mezclado o una muestra de pienso que no correspondió al momento en que se presentó el caso.

Este punto parece ser crítico a la hora de evaluar la concentración de $\mathrm{MN}$, dado que el proceso analítico mostró muy buena respuesta en la prueba de calibración. En establecimientos de gran envergadura, las 
dietas son preparadas diariamente en el mixer (carro de mezclado del alimento), por lo cual la muestra tomada para el análisis, puede tener una composición tal vez diferente a la que generó el caso de intoxicación, arrojando un resultado falso negativo. Asimismo, debe tenerse en cuenta que cuando aparecen los signos de intoxicación y/o muertes, muchas veces son causados por alimentos administrados horas o días previos al momento del muestreo.

Los ensayos preliminares indican que esta técnica es útil para la detección de $\mathrm{MN}$ en piensos, aunque no para otras formulaciones como pre-mezclas y alimento balanceado, considerando que ciertos componentes del alimento podrían generar un efecto matriz que dificulte la interpretación de los resultados. Del presente trabajo surge la necesidad de evaluar un mayor número de muestras, así como la necesidad de diseñar y aplicar muestreos representativos del comedero que uniformicen tanto la cantidad como la calidad de las muestras, para obtener alícuotas representativas de la partida de alimento problema.

A manera de conclusión es dable resaltar que la técnica ensayada en piensos se reveló útil para evaluar $\mathrm{MN}$ tanto en dosis terapéuticas como en sobredosificaciones. Aunque requiere cierta laboriosidad, es un método de bajo costo en cuanto a insumos y equipamientos requeridos, comparado con otros procedimientos. Su aplicación permitirá optimizar la formulación de las dietas y la concentración de sus componentes, evitando trastornos clínicos y subclínicos capaces de afectar la rentabilidad del sistema ganadero. Asímismo, tal práctica contribuiría a regular el uso racional de los productos farmacológicos de uso veterinario.

\section{REFERENCIAS}

1. Bogaert C, Jouany JP, Jeminet G. 1990. Effects of the ionophore antibiotics monensin, monensin-propionate, abierixin and calcimycin on ruminal fermentations in vitro. Anim Feed Sci Technol 28: 183-197.
2. Bretschneider G. 2009. Beneficios del uso de monensina en la alimentación del ganado para carne, leche y cría. Rev Electr Vet ISSN 1695-7504, vol. 10, № 10.

3. Dusi G, Gamba V. 1999. Liquid chromatography with ultraviolet detection of lasalocid, monensin, salinomycin and narasin in poultry feeds using pre-column derivatization. J Chromat A, 835: 243-246.

4. Food and Agricultural Materials Inspection Center (FAMIC). 2013. Manual sobre métodos de determinación de monensina sódica. Disponible on line: www.famic. go.jp/ffis/oie/obj/a28_mn.pdf, acceso 20-04-2013.

5. Greenfield H, Southgate DA. 2006. Datos de composición de alimentos: obtención, gestión y utilización, Public. FAO, Roma, $2^{\text {da }}$ ed., p. 238.

6. Nebot C, Iglesias A, Regal P, Miranda JM, Fente C, Cepeda A. 2012. A sensitive and validated HPLC-MS/ MS method for simultaneous determination of seven coccidiostats in bovine whole milk. Food Control 27: 29-36.

7. Odriozola N. 2004. Intoxicación por monensina. Diagnóstico Veterinario Especializado. INTA EEA Balcarce, Argentina. Disponible on line: http://www. produccionanimal.com.ar/sanidad_intoxicaciones_metabolicos/ intoxicaciones/41-i ntoxicacion_por_monensina.pdf]. Acceso: 24-09-15.

8. Pascuet ML, Moore DP, Iraguen I, Cosentino IA, Odriozola E. 2005. Cambios enzimáticos, lesiones macroscópicas y microscópicas producidas por la intoxicación con monensina en bovinos. Rev Med Vet 86: 47-51.

9. Redostits OM, Gay CC, Blood DC, Hinchcliff KW. 2002. Medicina Veterinaria (Capítulo: Intoxicación por ionóforos), $9^{\circ}$ ed., McGraw-Hill, Madrid, p.1931.

10. Rodríguez Armesto R, Peralta C, Ochoteco M, Picco E, Litterio N, Boggio JC. 2004. Posible intoxicación accidental con monensina en terneros destetados. Vet Arg 201: $13-20$.

11. Vickovic I, Sostaric B, Toncic J. 2010. Monensin toxicity and acute lethal rhabdomyolysis in accidental exposure in cattle (Abstracts of the XII International Congress of Toxicology), Toxicol Lett 196: 17, pag. S233. 